\title{
Tomato SIRbohB, a member of the NADPH oxidase family, is required for disease resistance against Botrytis cinerea and tolerance to drought stress
}

\author{
Xiaohui Li, Huijuan Zhang, Limei Tian, Lei Huang, Shixia Liu, Dayong Li* and \\ Fengming Song
}

National Key Laboratory for Rice Biology, Institute of Biotechnology, Zhejiang University, Hangzhou, China

\section{OPEN ACCESS}

Edited by: Jean-Michel Ané, University of Wisconsin-Madison,

USA

Reviewed by: Tesfaye Mengiste, Purdue University, USA

Kemal Kazan

Commonwealth Scientific and Industrial Research Organization,

Australia

*Correspondence:

Dayong Li,

National Key Laboratory for Rice Biology, Institute of Biotechnology, Zhejiang University, 866 Yuhangtang Road, Hangzhou, Zhejiang 310058,

China

dyli@zju.edu.cn

Specialty section: This article was submitted to Plant Biotic Interactions, a section of the journal Frontiers in Plant Science

Received: 17 April 2015 Accepted: 11 June 2015

Published: 23 June 2015

Citation:

Li X, Zhang H, Tian L, Huang L, Liu S, Li D and Song $F$ (2015) Tomato SIRbohB, a member of the NADPH oxidase family, is required for disease resistance against Botrytis cinerea and tolerance to drought stress.

Front. Plant Sci. 6:463. doi: 10.3389/fpls.2015.00463
NADPH oxidases (also known as respiratory burst oxidase homologs, Rbohs) are key enzymes that catalyze the generation of reactive oxygen species (ROS) in plants. In the present study, eight SIRboh genes were identified in tomato and their possible involvement in resistance to Botrytis cinerea and drought tolerance was examined. Expression of SIRbohs was induced by $B$. cinerea and Pseudomonas syringae pv. tomato but displayed distinct patterns. Virus-induced gene silencing based silencing of SIRbohB resulted in reduced resistance to $B$. cinerea but silencing of other SIRbohs did not affect the resistance. Compared to non-silenced plants, the SIRbohB-silenced plants accumulated more ROS and displayed attenuated expression of defense genes after infection with $B$. cinerea. Silencing of SIRbohB also suppressed flg22-induced ROS burst and the expression of S/Lrr22, a marker gene related to PAMP-triggered immunity (PTI). Transient expression of SIRbohB in Nicotiana benthamiana led to enhanced resistance to $B$. cinerea. Furthermore, silencing of $S / R b o h B$ resulted in decreased drought tolerance, accelerated water loss in leaves and the altered expression of drought-responsive genes. Our data demonstrate that SIRbohB positively regulates the resistance to $B$. cinerea, flg22-induced $\mathrm{PTI}$, and drought tolerance in tomato.

Keywords: tomato (Solanum lycopersicum L.), respiratory burst oxidase homologs (Rbohs), Botrytis cinerea, defense response, drought stress tolerance

\section{Introduction}

Reactive oxygen species (ROS) are known to participate in various cellular mechanisms and play multiple signaling roles in a wide range of organisms (Suzuki et al., 2011; Marino et al., 2012; Baxter et al., 2014; Kaur et al., 2014). Growing evidence indicates that ROS such as superoxide anion and hydrogen peroxide $\left(\mathrm{H}_{2} \mathrm{O}_{2}\right)$ are important signaling molecules that regulate a broad range of biological processes involved in growth, development, and responses to abiotic and biotic stresses (Torres et al., 2006; Torres, 2010; Mittler et al., 2011; Marino et al., 2012; Nathan and CunninghamBussel, 2013; Baxter et al., 2014; Kaur et al., 2014).

In plants, ROS are mainly generated by a number of enzymes (Apel and Hirt, 2004; Tripathy and Oelmuller, 2012). NADPH oxidases, also known as respiratory burst oxidase homologs 
(Rbohs), are the most extensively studied ROS-generating enzymes (Sagi and Fluhr, 2006; Suzuki et al., 2011). Rbohs are localized on the plasma membrane; however, dynamic changes in the subcellular localization of tobacco $\mathrm{NtRbohD}$ in response to elicitor treatment were recently reported (Noirot et al., 2014). Generally, Rbohs form enzymatic complexes and catalyze the production of superoxide radicals via FAD and two independent hemes (Sagi and Fluhr, 2006). It was suggested that plant and animal Rbohs share a common mechanism for activation of their enzymatic activity upon developmental and stress signals (Canton and Grinstein, 2014). The activity of plant Rbohs was found to be regulated in different ways, e.g., the binding of Rac GTPase to the N-terminal extension (Wong et al., 2007; Oda et al., 2010), protein modification via phosphorylation (Xing et al., 2001; Kobayashi et al., 2007; Ogasawara et al., 2008; Sirichandra et al., 2009; Yoshioka et al., 2009; Kimura et al., 2012; Takahashi et al., 2012; Dubiella et al., 2013), S-nitrosylation (Yun et al., 2011), and extracellular ATP and phospholipid signaling (Song et al., 2006; Demidchik et al., 2009; Zhang et al., 2009).

Plant Rbohs constitute a multigene family and have been identified in a wide range of plant species (Kaur et al., 2014). The Rboh family is comprised of 10 members in Arabidopsis, named AtRbohA-H, and members in rice (Sagi and Fluhr, 2006; Wong et al., 2007). Recent studies have revealed that plant Rbohs are involved in a multitude of different signaling pathways that regulate root hair growth, stomatal closure, pollen-stigma interactions, defense responses to pathogens, and acclimation to abiotic stresses (Torres, 2010; Suzuki et al., 2011; Marino et al., 2012; Nathan and Cunningham-Bussel, 2013; Baxter et al., 2014; Kaur et al., 2014). In Arabidopsis, AtRbohE was found to regulate the proper timing of tapetal-programmed cell death, a process that is critical for pollen development (Xie et al., 2014), and AtRbohH and AtRbohJ have been shown to modulate the amplitude and frequency of pollen tube growth and seed development (Müller et al., 2009; Kaya et al., 2014; Lassig et al., 2014). It was also found that the Arabidopsis AtRbohD, AtRbohF, and AtRbohC, maize Roothairless5 and cress LesaRbohB play roles in regulating lateral root development (Foreman et al., 2003; Jones et al., 2007; Macpherson et al., 2008; Muller et al., 2012; Jiao et al., 2013; Nestler et al., 2014; Li et al., 2015). The bean PvRbohB and Medicago truncatula MtRbohA were found to positively regulate nitrogen fixation and delay nodule senescence but negatively regulate AM colonization (Marino et al., 2011; Montiel et al., 2012; Arthikala et al., 2013, 2014). In addition to the functions in growth and development, Rbohs have been shown to play important roles in plant abiotic and biotic stress responses. The requirements for Rbohs in defense responses against different pathogens seem to be diverse in plant-pathogen interactions (Pogány et al., 2009; Torres et al., 2013). In Arabidopsis, AtRbohD and AtRbohF are required for the accumulation of ROS and function as critical regulators of defense responses (Torres et al., 2002, 2013; Maruta et al., 2011; Chaouch et al., 2012; Foley et al., 2013; Nozaki et al., 2013; Pastor et al., 2013). Although silencing of AtRbohB eliminated elicitin- and MAPK-mediated ROS generation (Asai et al., 2008), AtRbohD-regulated ROS burst is not linked to MPK3/MPK6 activation during early signaling events in plant immunity (Xu et al., 2014). In barley, silencing of $H v R b o h A$ or knockdown of $\mathrm{HvRbohF2}$ affected penetration by Blumeria graminis f. sp. hordei (Trujillo et al., 2006; Proels et al., 2010). The tobacco $N t R b o h D$ is responsible for ROS production in cryptogeinelicited cells and in herbivore-elicited responses (Simon-Plas et al., 2002; Lherminier et al., 2009; Wu et al., 2013), whereas silencing of NbRbohA and NbRbohB in Nicotiana benthamiana resulted in reduced ROS production in response to Phytophthora infestans (Yoshioka et al., 2003). Furthermoe, AtRbohD and AtRbohF were found to participate in ABA and ethylene signaling resulting in stomatal closure (Kwak et al., 2003; Bright et al., 2006; Desikan et al., 2006) and both of them have been shown to mediate rapid signaling that regulates abiotic stress responses (Miller et al., 2009; Xie et al., 2011; Ma et al., 2012).

Two Rboh genes, SlRboh1 and SlWfil, have been identified in tomato. These genes have been shown to be involved in wounding responses and development (Sagi et al., 2004). Recent studies revealed that SlRboh1 is required for brassinosteroid-induced apoplastic $\mathrm{H}_{2} \mathrm{O}_{2}$ production and stomatal closure/opening. Therefore, SlRboh1 plays an important role in acclimationinduced stress cross-tolerance (Zhou et al., 2012, 2014; Xia et al., 2014). However, the SlRboh family and the function of SlRbohs in biotic and abiotic stress responses are largely unknown. In the present study, we characterized the SlRboh family in tomato and investigated the possible involvement of SlRbohs in disease resistance and drought stress tolerance using a virus-induced gene silencing (VIGS) approach. Our VIGSbased functional analyses demonstrate that SlRbohB positively regulates the defense response against Botrytis cinerea, the flg22-induced immune response and tolerance to drought stress.

\section{Materials and Methods}

\section{Plant Growth, Treatments, and Pathogen Inoculation}

Tomato (Solanum lycopersicum) cv. Suhong 2003 was used for all experiments. Seedlings were grown in a mixture of perlite: vermiculite: plant ash (1:6:2) in a growth room under fluorescent light $\left(200 \mu \mathrm{E} \mathrm{m} \mathrm{m}^{2} \mathrm{~s}^{-1}\right)$ at $22-24^{\circ} \mathrm{C}$ with $60 \%$ relative humidity and a $14 \mathrm{~h}$ light/10 h dark cycle. Pathogen inoculation, disease assays with $B$. cinerea or Pseudomonas syringae pv. tomato (Pst) DC3000 and the measurement of in planta fungal growth were basically performed according to previously described protocols (AbuQamar et al., 2008; Li et al., 2014a). Drought stress was applied to the plants by withholding watering for 2 weeks and stress phenotypes were recorded and photographed. For the measurement of water loss, fully expanded leaves were detached and water loss was measured according to a previously described method (Liu et al., 2014). Leaf samples were collected at the indicated time points after treatment or inoculation and used immediately for physiological and biochemical analyses or stored at $-80^{\circ} \mathrm{C}$ until use. 


\section{Identification of SIRboh Genes and Bioinformatics Analysis}

Arabidopsis AtRbohs were used as queries to search against the tomato genomic database at the SOL Genomics Network (SGN) ${ }^{1}$. The obtained candidate SlRboh sequences were examined using the domain analysis programs PFAM $^{2}$ and SMART $^{3}$ with the default cutoff parameters. The Arabidopsis, rice, and tomato Rboh protein sequences were aligned using the multiple alignment program MUSCLE 3.8.31 (Edgar, 2004). Maximum likelihood (ML) analyses were carried out using RAxML-HPC v.8 (Stamatakis, 2006; Stamatakis et al., 2008) on the XSEDE Teragrid of the CIPRES science Gateway (Miller et al., 2010) with default settings and JTT, followed by 1000 bootstrap replicates.

\section{Construction of VIGS and Transient Expression Constructs}

For the VIGS constructs, fragments of 300-400 bp in sizes for SlRbohs were amplified from tomato cDNA using genespecific primers (Supplementary Table S1), sequenced and then cloned into the TRV2 vector (Liu et al., 2002), yielding TRV2SlRbohA-H. For the transient expression constructs, the SlRbohB coding sequence was amplified using the primers SIRbohBGFP-F and SlRbohB-GFP-R (Supplementary Table S1) and cloned into pFGC-Egfp at XbaI/SmaI sites, yielding pFGCSlRbohB. The recombinant plasmids TRV2-SlRbohA-H and pFGC-SlRbohB were introduced into Agrobacterium tumefaciens strain GV3101 by electroporation using the GENE PULSER II Electroporation System (Bio-Rad Laboratories, Hercules, CA, USA). Agrobacteria carrying different plasmids (TRV2SlRbohA-H or pFGC-SlRbohB) were grown in YEP medium (50 $\mu \mathrm{g} \mathrm{ml}^{-1}$ rifampicin, $50 \mu \mathrm{g} \mathrm{ml}^{-1}$ kanamycin, and $25 \mu \mathrm{g} \mathrm{ml}^{-1}$ gentamicin) for $24 \mathrm{~h}$ with continuous shaking at $28^{\circ} \mathrm{C}$. Cells were collected by centrifugation and resuspended in infiltration buffer (10 $\mathrm{mM} \mathrm{MgCl} 2,10 \mathrm{mM} \mathrm{MES}$, and $200 \mu \mathrm{M}$ acetosyringone at $\mathrm{pH} 5.7)$.

\section{Agroinfiltration for VIGS and Transient Expression}

For the VIGS assays, agrobacteria harboring TRV2-SlRbohA-H were mixed with agrobacteria carrying TRV1 at a ratio of $1: 1$ and adjusted to $\mathrm{OD}_{600}=1.5$. The mixed agrobacteria suspensions were infiltrated separately into the abaxial surface of 2-week-old seedlings using $1 \mathrm{ml}$ needleless syringes (Liu et al., 2002). The VIGS-infiltrated plants were allowed to grow for 3 weeks before use in all experiments. For transient expression in $N$. benthamiana, agrobacteria carrying pFGC-SlRbohB or pFGC-eGFP empty vector were infiltrated into leaves of 4week-old plants using $1 \mathrm{ml}$ needleless syringes. Leaf samples were collected for analyzing the expression level of SlRbohB and for disease phenotyping and physiological, biochemical, and molecular analyses.

\footnotetext{
${ }^{1}$ http://solgenomics.net

${ }^{2}$ http://pfam.sanger.ac.uk/

${ }^{3}$ http://smart.embl-heidelberg.de/
}

\section{qRT-PCR Analysis of Gene Expression}

Total RNA was extracted using Trizol regent (TAKARA, Dalian, China) and treated with RNase-free DNase according to the manufacturer's instructions. First strand cDNA was synthesized by reverse transcription using PrimeScript RT regent kit (TAKARA, Dalian, China) and the obtained cDNAs were used for gene expression analysis with qRT-PCR. Each qPCR reaction contained 12.5 $\mu \mathrm{L}$ SYBR Premix Ex TaqTM (TAKARA, Dalian, China), $0.1 \mu \mathrm{g}$ cDNA and $7.5 \mathrm{pmol}$ of each genespecific primer (Supplementary Table S1) in a final volume of $25 \mu \mathrm{L}$, and performed on a CFX96 real-time PCR system (BioRad, Hercules, CA, USA). Relative gene expression levels were calculated using $2^{-\Delta \Delta C T}$ method (Livak and Schmittgen, 2001). Three independent biological replicates were analyzed.

\section{Western Blot Analysis}

Extraction of total proteins from leaf samples, separation on SDS-PAGE gels and wet electroblotting transferring onto nitrocellulose membranes were carried out according to previously described protocols (Li et al., 2014a). GFP was detected using a mouse monoclonal GFP antibody (1:1000 dilution; No. M1210-1, Huaan Company, Hangzhou, China) and a peroxidase-conjugated anti-mouse antibody (1:8000 dilution; No. HA1008, Huaan Company, Hangzhou, China) according to the manufacturer's instructions. Proteins on PVDF membranes were detected by SuperSignal West Pico Chemiluminescent Substrate (Pierce, Rockford, IL, USA).

\section{In Situ Staining and Measurement of $\mathrm{H}_{2} \mathrm{O}_{2}$}

In situ staining of $\mathrm{H}_{2} \mathrm{O}_{2}$ was performed by 3, 3-diaminobenzidine (DAB) staining (Thordal-Christensen et al., 1997; Li et al., 2014a). The accumulation of $\mathrm{H}_{2} \mathrm{O}_{2}$ in stained leaves was visualized using a digital camera. Measurement of ROS burst in leaves was performed using a luminol-based luminescence method (Chakravarthy et al., 2010). Briefly, 4-mm leaf disks were floated

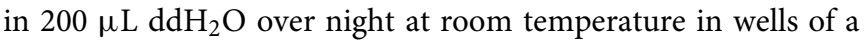
96-well plate. Disks were then placed in $100 \mu \mathrm{L} 400 \mathrm{nM}$ flg22 solution containing $34 \mu \mathrm{g} / \mathrm{ml}$ of luminol (Sigma, St. Louis, MO, USA) and $20 \mu \mathrm{g}$ of horseradish peroxidase (VI-A, Sigma, St. Louis, MO, USA) or in solution as a control. Luminescence was measured continuously at $1 \mathrm{~min}$ intervals for $30 \mathrm{~min}$ using a Synergy HT plate reader (BioTek Instruments, Inc. Winooski, VT, USA). Three replications were performed for each treatment.

\section{Statistical Analysis}

All experiments were repeated independently three times. Data obtained from three independent experiments were subjected to statistical analysis according to Student's $t$-test. Probability values of $p<0.05$ were considered to represent significant differences.

\section{Results}

\section{Identification of the SIRbohs Family in Tomato}

Blastp searches against the tomato gnomic database using Arabidopsis AtRboh proteins as reference queries obtained nine significant hits corresponding to non-redundant putative 
TABLE 1 | Characterization of tomato NADPH oxidase genes.

\begin{tabular}{|c|c|c|c|c|c|c|c|}
\hline Genes & Other names & Locus ID in SOL & Proteins in NCBI & cDNAs in SOL and NCBI & Size (aa) & MW (kD) & pl \\
\hline SIRbohA & & Solyc01g099620 & XM_004230184 & SGN-U586275, SGN-U601117 & 865 & 98.73 & 8.79 \\
\hline SIRbohB & SIWfi1 & Solyc03g117980 & NM_001247342 & SGN-U579691, AF148534 & 938 & 105.31 & 9.08 \\
\hline SIRbohC & & $\begin{array}{l}\text { Solyc05g025690+ } \\
\text { Solyc05g025680a }\end{array}$ & XM_004239534 & NA & 866 & 98.71 & 8.81 \\
\hline SIRbohD & & Solyc06g068680 & XM_004241593 & SGN-U567947, SGN-U577640 & 857 & 97.73 & 9.01 \\
\hline SIRbohE & & Solyc06g075570 & XM_004242033 & NA & 830 & 95.40 & 9.02 \\
\hline SIRbohF & & Solyc07g042460 & XM_006353710 & SGN-U573231, SGN-U575883 & 881 & 100.06 & 9.03 \\
\hline SIRbohG & SIRboh1 & Solyc08g081690 & NM_001288375 & SGN-U564615, AF088276 & 963 & 109.08 & 9.09 \\
\hline SIRbohH & & Solyc11g072800 & XM_004251404 & SGN-U572889 & 816 & 93.67 & 8.87 \\
\hline
\end{tabular}

a The predicted loci Solyc05g025690 and Solyc05g025680 are a single gene coding for SIRbohC.

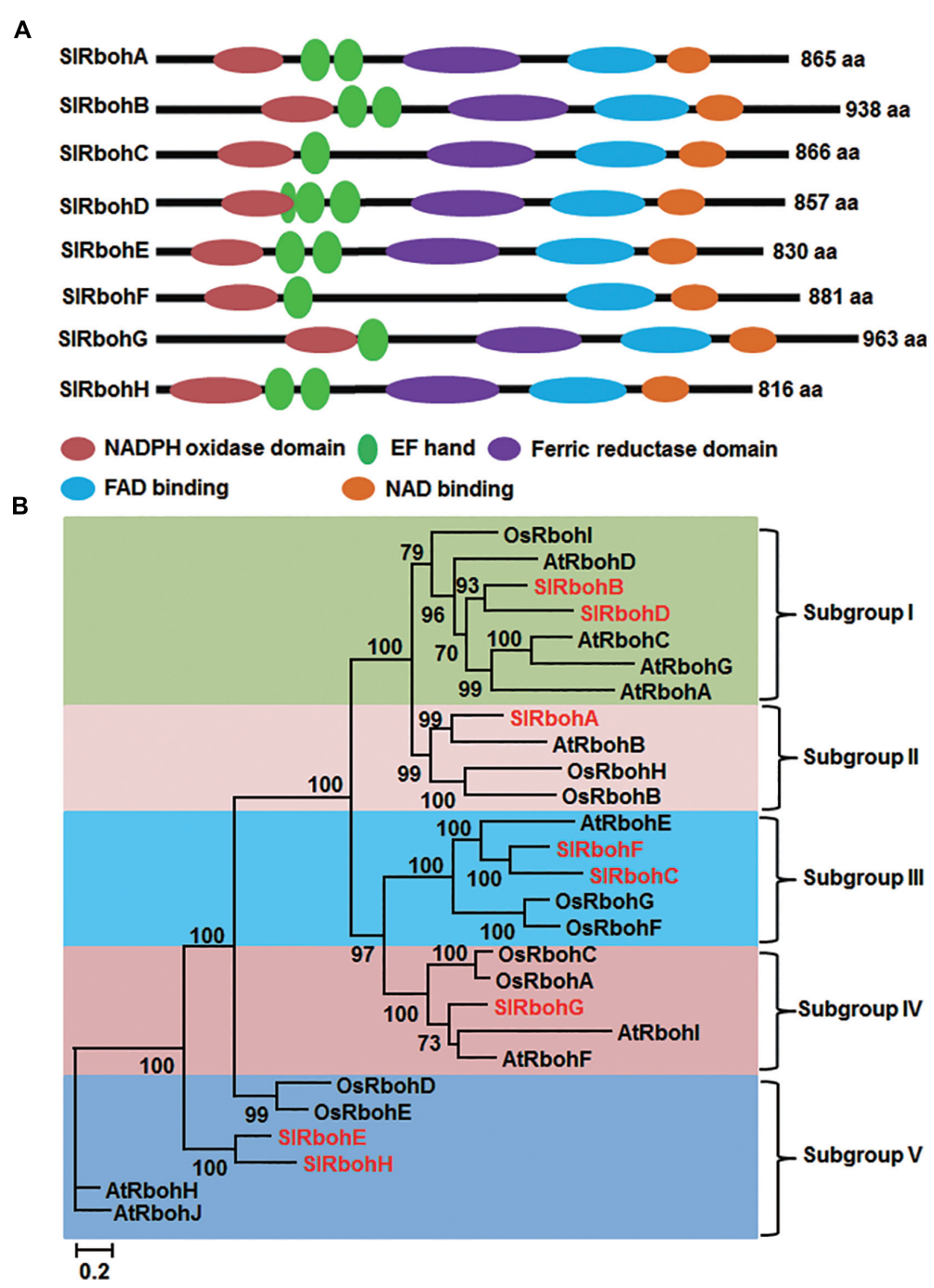

FIGURE 1 | Structure and phylogenetic tree of tomato Rboh proteins.

(A) Functional domains and their organization in SIRboh proteins.

(B) Maximum-likelihood (ML) tree of SIRbohs with Arabidopsis AtRbohs and rice
OsRbohs. The SIRboh proteins are indicated with red color. Only bootstrap values $>50 \%$ are shown, and branch lengths are proportional to the number of substitutions per site (see scale bars). 
SlRboh genes (Table 1). The predicted loci Solyc05g025680 and Solyc05g025690 are indeed the same gene, which encodes for SlRbohC. The SlRboh family therefore contains eight members, named SlRbohA-H (Table 1). SlRbohB and SlRbohG were previously reported as $S l W f i 1$ and $S l R b o h 1$, respectively (Sagi et al., 2004). By searching the SOL UniGene and NCBI databases, six out of eight SlRboh genes have full-length cDNA supports (Table 1). The sizes of the SlRboh proteins range from 816 (SlRbohH) to 963 (SlRbohG) amino acids with molecular weights of 94-109 kD and pIs of 8.63-9.09 (Table 1). Although the SlRboh proteins vary in size, they all share major functional domains with similar organization (Figure 1A); however, SlRbohF lacks the ferric reductase domain. The SlRboh proteins contain a conserved NADPH oxidase domain, 1-3 EF-hand domains, a ferric reductase domain, an FAD binding domain, and a NAD binding domain (Figure 1A). Phylogenetic analyses with Arabidopsis AtRbohs and rice OsRbohs indicate that SlRbohs can be classified into five subgroups (I-V; Figure 1B). Notably, each subgroup contains Rbohs from tomato, Arabidopsis, and rice. In the phylogenetic tree, SIRbohB and SIRbohD, with AtRbohA/C/D/G and OsRbohI, are assigned to subgroup I.
SlRbohA, together with AtRbohB, OsRbohB/H, is classified into subgroup II. SIRbohC and SIRbohF are members of subgroup III, which contains AtRbohE and OsRbohF/G. SlRbohG belongs to subgroup VI, which includes AtRbohI/F and OsRbohA/C. The remaining proteins SlRbohE and SIRbohH, together with AtRbohJ/H and OsRbohE/D, are classified into subgroup V.

\section{Expression of SIRbohs in Response to B. cinerea and Pst DC3000}

To explore the possible involvement of SlRbohs in the defense response against pathogen, we analyzed and compared the expression patterns of SlRbohs in tomato plants after infection with $B$. cinerea, a necrotrophic fungal pathogen causing gray mold disease, or Pst DC3000, a (hemi)biotrophic bacterial pathogen causing bacterial leaf spot disease. To confirm the efficiency of the inoculation procedure, the expression patterns of SlLapA, a defense gene regulated by the JA/ET-mediated signaling pathway that is involved in the defense response against $B$. cinerea, and $S I P R-P 2$, a defense gene regulated by the SA signaling pathway that is involved in the defense response against Pst DC3000, were monitored. As shown in Figure 2,

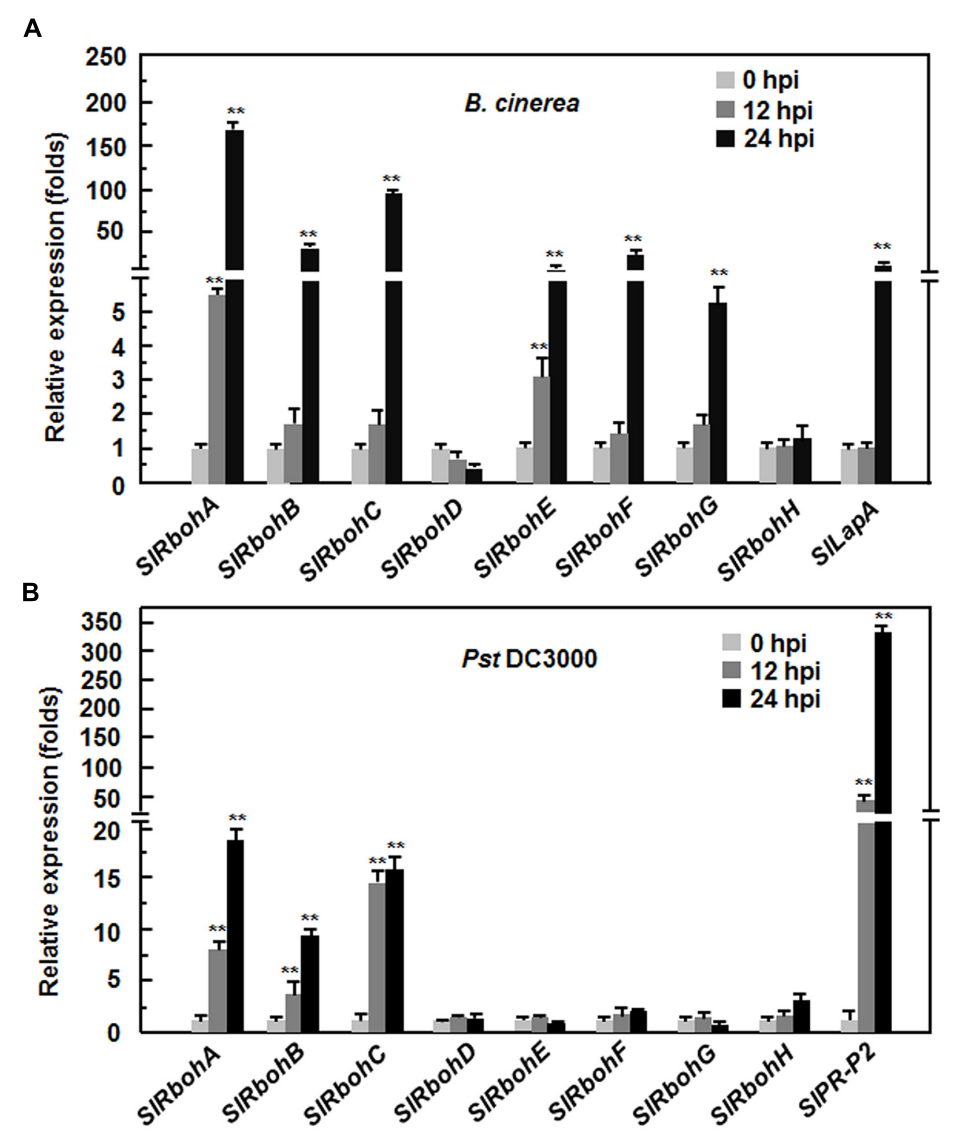

FIGURE 2 | Expression patterns of SIRbohs in response to Botrytis cinerea and Pst DC3000. Tomato plants were inoculated by foliar spraying with spore suspension $\left(2 \times 10^{5}\right.$ spores $\left./ \mathrm{mL}\right)$ of $B$. cinerea (A) or vacuum infiltration with Pseudomonas syringae pv. tomato DC3000

$\left(\mathrm{OD}_{600}=0.0002\right)$, (B) and leaf samples were collected at indicated time points. Gene expression was analyzed by qRT-PCR and relative expression levels were calculated by comparing with the corresponding values at $\mathrm{O} \mathrm{h}$ (as a control) after inoculation. Data presented are the means \pm SD from three independent experiments and ${ }^{* *}$ above the columns indicate significant differences at $p<0.05$ level. 
the expression level of SlLapA in B. cinerea-inoculated plants showed a $>40$-fold increase at 24 hours post inoculation (hpi) while the expression level of SlPR-P2 in Pst DC3000-inoculated plants displayed $\sim 50$-fold and $>300$-fold increases at 12 and $24 \mathrm{hpi}$, respectively. These findings indicate that the effectiveness of the inoculation procedure was satisfactory for further analysis of the expression patterns of SlRbohs in response to B. cinerea and Pst DC3000. After infection with $B$. cinerea, the expression of SlRbohA, SlRbohB, SlRbohC, SlRbohE, SlRbohF, and SlRbohG was significantly induced; the expression of SlRbohD and SlRbohH was not affected (Figure 2A). Among the $B$. cinerea-inducible SlRbohs, the expression levels of SlRbohA and SlRbohE started to increase at $12 \mathrm{hpi}$ and peaked at $48 \mathrm{hpi}$, whereas the expression levels of SlRbohB, SlRbohC, SlRbohF, and SlRbohG only increased significantly at $24 \mathrm{hpi}$ after infection with $B$. cinerea, as compared with those in the mock-inoculated plants (Figure 2A). After infection with Pst DC3000, the expression of SlRbohD, SlRbohE, SlRbohF, SlRbohG, and SlRbohH was not affected; the expression levels of SlRbohA, SlRbohB, and SlRbohC were significantly increased at 12 and 24 hpi compared to mock-inoculated plants (Figure 2B). These results indicate that SlRboh genes respond differentially to B. cinerea and Pst DC3000, showing different dynamics and magnitude of expression after pathogen infection.

\section{Silencing of SIRbohB Resulted in Reduced Resistance to $B$. cinerea}

Considering that relatively little is known about the function of Rbohs in resistance to necrotrophic fungal pathogens, we focused our efforts on exploring the possible involvement of SlRbohs in resistance to $B$. cinerea through VIGS-based functional analyses. For this purpose, standard VIGS procedure with a TRV2-PDS construct as an indicative for VIGS efficiency of each experiment was performed on 2-week-old tomato plants (Liu et al., 2002; Li et al., 2014a), followed by disease assays with $B$. cinerea at 4 weeks after VIGS infiltration. Under our experimental conditions, $\sim 90 \%$ of TRV2-PDS-infiltrated plants showed a bleaching phenotype. Silencing efficiency for each SlRboh gene was evaluated by qRT-PCR and the transcript levels of the target SlRboh genes in the TRV2-SlRboh-infiltrated plants were compared to that in the TRV2-GUS-infiltrated plants. As shown in Figure 3, the transcript levels of the target SlRboh genes in the corresponding TRV2-SlRboh-infiltrated plants were significantly reduced and the silencing efficiency for the SlRboh genes was estimated to be $65-70 \%$, indicating that the silencing efficiency with the designed constructs for each SlRboh gene under our VIGS procedure was appropriate for further experiments.

Next, we analyzed the resistance of the SlRboh-silenced plants to $B$. cinerea using a detached leaf inoculation assay. The lesions on leaves from TRV2-SlRbohA-, TRV2-SlRbohC-, TRV2SlRbohD-, TRV2-SlRbohE-, TRV2-SlRbohF-, TRV2-SlRbohG-, or TRV2-SlRbohH-infiltrated plants were comparable to those from TRV2-GUS-infiltrated plants and wild type (WT) plants (Figures 4A,B), whereas the lesions on leaves from TRV2SlRbohB-infiltrated plants were significantly larger at 3 days post inoculation (dpi; Figure 4A), showing an approximately $40 \%$ larger in size than those in TRV2-GUS-infiltrated plants or WT plants (Figure 4B). To confirm the disease phenotype observed

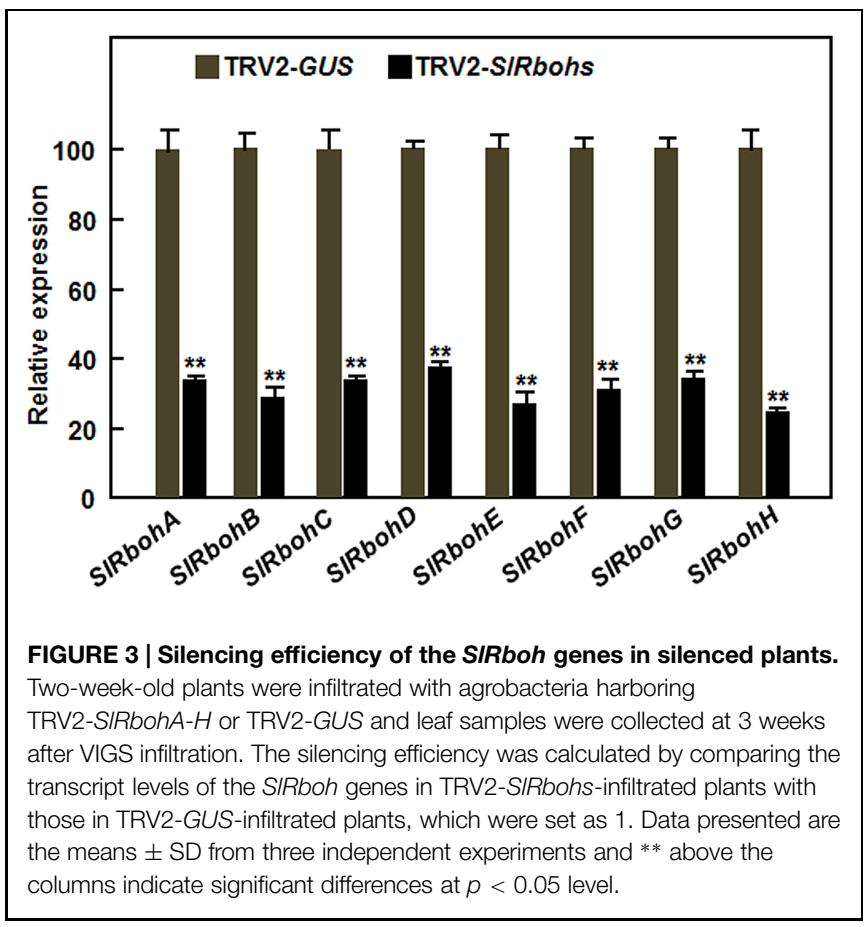

in TRV2-SlRbohB-infiltrated plants, the in planta growth of $B$. cinerea was measured by analysis of the transcript level of the actin gene BcActin as an indicator of the rate of fungal growth and compared between the TRV-SlRbohB- and TRVGUS-infiltrated plants in whole plant inoculation assays. As shown in Figure 4C, the in planta growth of $B$. cinerea in TRV2-SlRbohB-infiltrated plants increased markedly at 2 and $3 \mathrm{dpi}$, leading to three times greater than that observed in TRV2-GUS-infiltrated plants at $3 \mathrm{dpi}$. Together, these data demonstrate that silencing of $\operatorname{SlR} b o h B$ attenuated the resistance to $B$. cinerea and $S l R b o h B$ is therefore required for resistance against B. cinerea whereas SlRbohA, SlRbohC, SlRbohD, SlRbohE, SlRbohF, SlRbohG, and SlRbohH may not be involved in resistance to $B$. cinerea.

\section{Transient Expression of SIRbohB in $N$. benthamiana Conferred Increased Resistance to B. cinerea}

To further confirm the function of SlRbohB in resistance to $B$. cinerea, we examined whether overexpression of $S l R b o h B$ could confer increased resistance to $B$. cinerea. When transiently expressed in $N$. benthamiana leaves, high levels of SlRbohB expression and the SIRbohB-GFP fusion protein were detected (Figures 5A,B). In disease assays, the lesions on leaves of SlRbohB-infiltrated $N$. benthamiana plants were significantly smaller than those on eGFP vector-infiltrated control plants (Figure 5C), leading to approximately $40 \%$ of reduction in lesion size, at $5 \mathrm{dpi}$ (Figure 5D). These data demonstrate that transient expression of $S l R b o h B$ in $N$. benthamiana conferred an increased resistance to $B$. cinerea. Therefore, SlRbohB positively regulates the defense response against B. cinerea. 


\section{A}

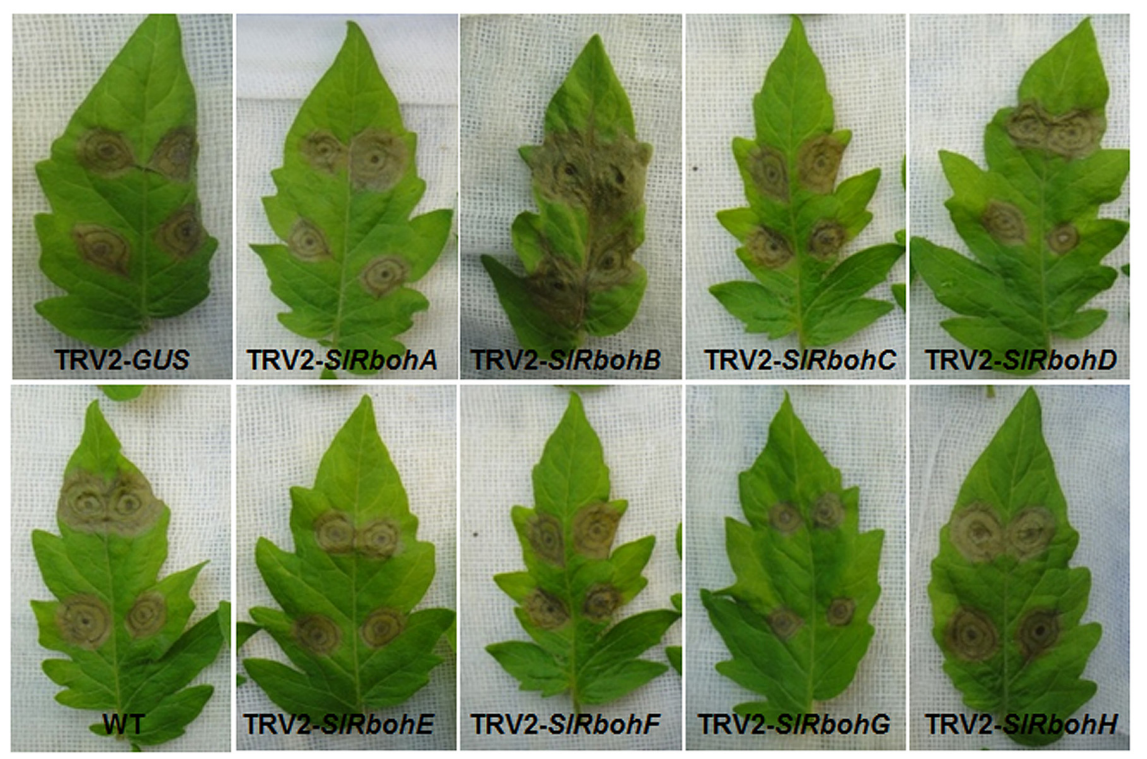

B

C
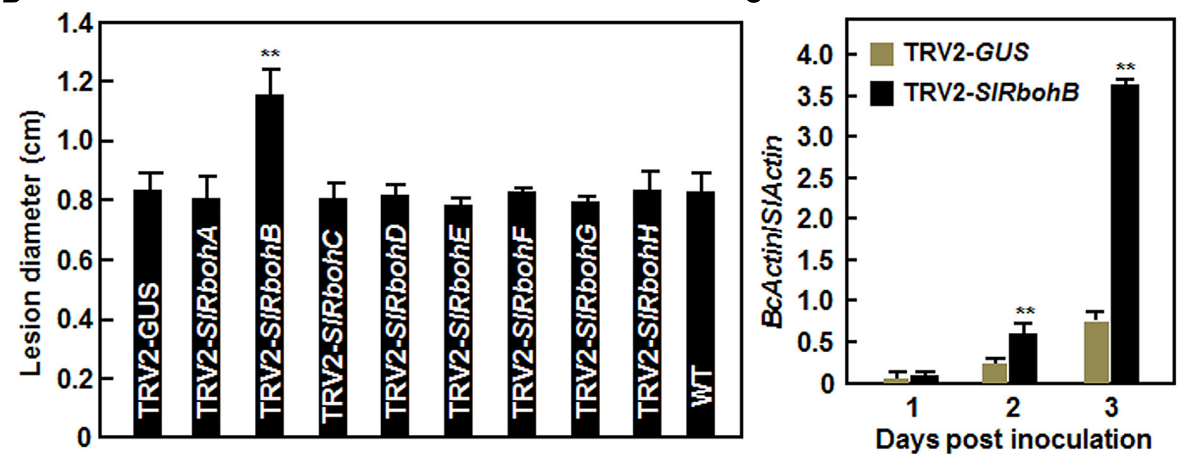

FIGURE 4 | Silencing of SIRbohB led to reduced resistance to B. cinerea. (A) Disease symptom and (B) lesion size. Two-week-old plants were infiltrated with agrobacteria harboring TRV2-SIRbohA-H or TRV2-GUS and leaves were detached for disease assays at 4 weeks after VIGS infiltration. Inoculation was done by dropping spore suspension $\left(1 \times 10^{5}\right.$ spores $\left./ \mathrm{mL}\right)$ on detached leaves and lesion sizes were measured at 4 days after inoculation. A minimum of 20 leaves were included in each experiment. (C) In planta growth of $B$. cinerea in inoculated leaves. Fungal growth was assumed by analyzing the transcript level of BcActin gene by qRT-PCR using SIActin gene as an internal control and shown as the ratios of BcActin/SIActin. Data presented in $(\mathbf{B}, \mathbf{C})$ are the mean \pm SD from three independent experiments and ${ }^{* *}$ above the columns indicate significant differences at $p<0.05$ level.

\section{Silencing of SIRbohB Attenuated Defense Response to $B$. cinerea}

To explore the possible mechanism involved in the reduced resistance observed in $S l R b o h B$-silenced plants, we analyzed and compared the accumulation of $\mathrm{H}_{2} \mathrm{O}_{2}$ and the expression of defense genes in TRV2-SlRbohB- and TRV2-GUS-infiltrated plants. No difference in the accumulation of $\mathrm{H}_{2} \mathrm{O}_{2}$, as detected by DAB staining, was detected in leaves of TRV2SlRbohB- and TRV2-GUS-infiltrated plants without B. cinerea infection (data not shown). A significant accumulation of $\mathrm{H}_{2} \mathrm{O}_{2}$, shown as brown precipitates in leaves, was detected in TRV2-SlRbohB- and TRV2-GUS-infiltrated plants after infection with $B$. cinerea (Figure 6A). However, the accumulation of $\mathrm{H}_{2} \mathrm{O}_{2}$ in the leaves of TRV2-SlRbohB-infiltrated plants was reduced compared with that of TRV2-GUS-infiltrated plants (Figure 6A). Conversely, the expression levels of selected defense genes increased significantly in TRV2-SlRbohB- and TRV2-GUS-infiltrated plants after infection with $B$. cinerea. However, the kinetic of expression exhibited distinct patterns (Figure 6B). After infection with $B$. cinerea, the expression levels of SIPRP1b, SILAPA, and SIPIN2 in TRV2-SlRbohBinfiltrated plants were comparable to those in TRV2-GUSinfiltrated plants at $1 \mathrm{dpi}$ but were significantly decreased at $2 \mathrm{dpi}$, by reduction of $50 \%$ for $S I P R 1 b$ and $>90 \%$ for SlLapA and SIPIN2 (Figure 6B). However, the expression level of SlPR$P 2$ in TRV2-SlRbohB-infiltrated plants was higher than that in TRV2-GUS-infiltrated plants at $1 \mathrm{dpi}$ but the expression levels were similar at $2 \mathrm{dpi}$ (Figure 6B). These results indicate that silencing of $S l R b o h B$ compromised the accumulation of $\mathrm{H}_{2} \mathrm{O}_{2}$ and attenuated the defense response by downregulating the expression of defense genes in tomato upon infection with B. cinerea. 

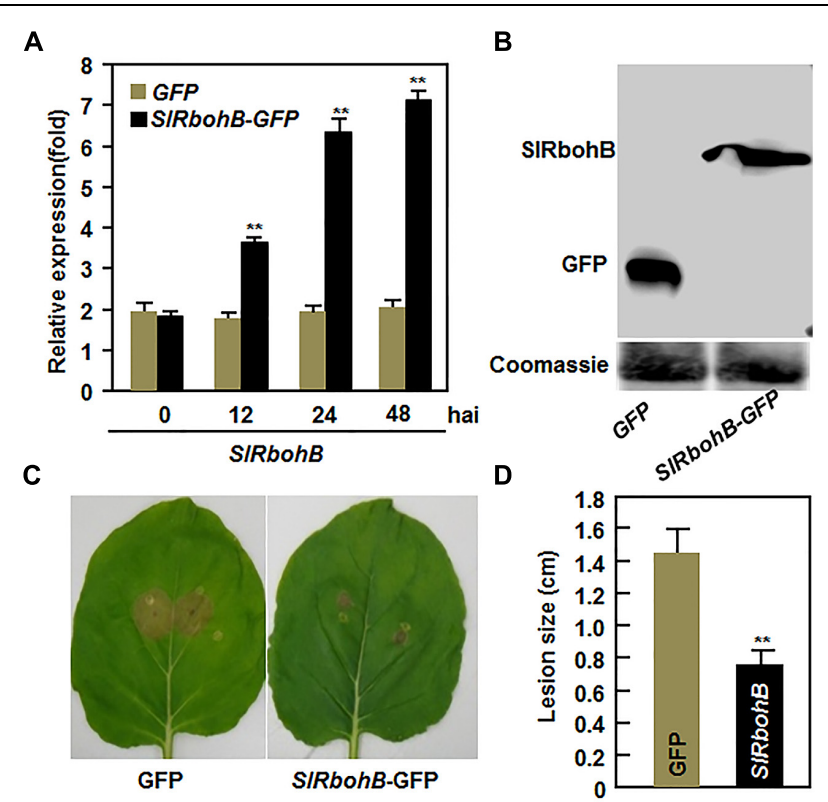

FIGURE 5 | Transient expression of SIRbohB in Nicotiana benthamiana conferred an increased resistance to $B$. cinerea. Agrobacteria harboring pFGC-SIRbohB or pFGC-eGFP were infiltrated into leaves of 4-week-old $N$. benthamiana plants and leaf samples were collected for different assays. (A) Expression of SIRbohB in pFGC-SIRbohB-infiltrated leaves. (B) The SIRbohB-GFP fusion protein in pFGC-SIRbohB-infiltrated leaves. Leaf samples were harvested $48 \mathrm{~h}$ after infiltration, total soluble proteins were separated by SDS-PAGE, and analyzed by immunoblotting using a GFP-specific antibody. Equal loading of total proteins was verified by Coomassie blue staining. (C) Disease symptom on representative leaves. (D) Lesion sizes. The pFGC-SIRbohB- and pFGC-eGFP-infiltrated leaves were detached at $48 \mathrm{~h}$ after infiltration and inoculated by dropping spore suspension $\left(2 \times 10^{5}\right.$ spores $/ \mathrm{mL}$ ). Photos were taken and lesion sizes were measured on a minimum of 10 leaves at 5 days after inoculation. Data presented in (A,D) are the mean \pm SD from three independent experiments and ${ }^{* *}$ above the columns indicate significant difference at $p<0.05$ level.

\section{Silencing of SIRbohB Attenuated flg22-Induced PAMP-Triggered Immunity (PTI)}

Several Arabidopsis AtRbohs have been shown to play important roles in PAMP-triggered immunity (PTI; Kaur et al., 2014). Thus, we explored whether SIRbohB has a function in PTI by analyzing and comparing the flg22-induced ROS burst and the expression of PTI marker genes in TRV2-SlRbohB- and TRV2GUS-infiltrated plants. As shown in Figure 7A, a significant ROS burst in the leaves of TRV2-GUS-infiltrated plants was detected within 4-18 min, while no such ROS burst was observed in the leaves of TRV2-SlRbohB-infiltrated plants after the addition of $400 \mathrm{nM}$ flg22. No ROS burst was observed in the untreated leaves of TRV2-GUS- and TRV2-SlRbohBinfiltrated plants (Figure 7A). The expression level of SlLrr22, a PTI marker gene in tomato (Taylor et al., 2012), in TRV2SlRbohB-infiltrated plants was significantly lower than that in TRV2-GUS-infiltrated plants at 2 and 6 hours after treatment (hat) with flg22 (Figure 7B). These data indicate that silencing of SlRbohB also attenuated the flg22-induced PTI, demonstrating a role for SlRbohB in PTI.

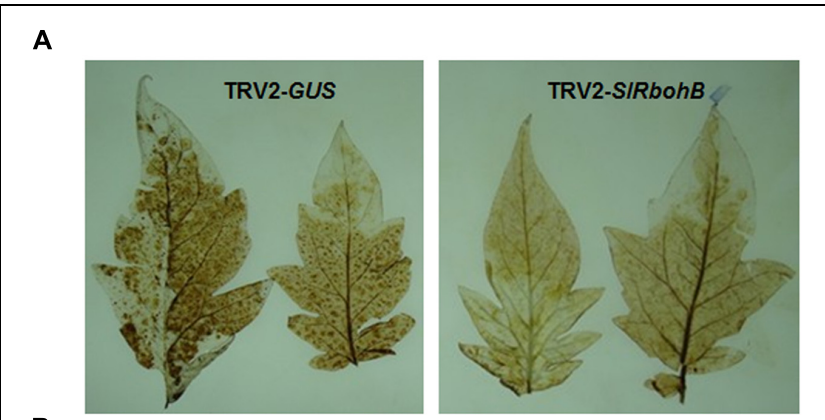

B

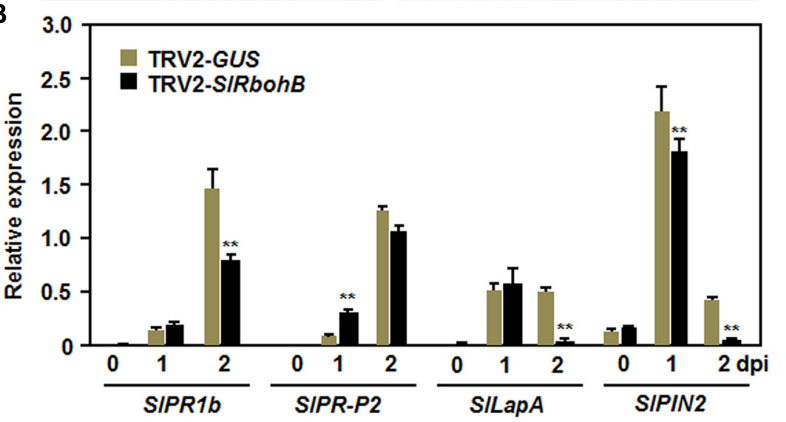

FIGURE 6 | Silencing of SIRbohB attenuated B. cinerea-induced defense response. Two-week-old plants were infiltrated with agrobacteria harboring TRV2-SIRbohB or TRV2-GUS and the leaves were inoculated by dropping spore suspension $\left(1 \times 10^{5}\right.$ spores $\left./ \mathrm{mL}\right)$ at 4 weeks after agroinfiltration. (A) Accumulation of $\mathrm{H}_{2} \mathrm{O}_{2}$ and (B) expression of the selected defense genes. Accumulation of $\mathrm{H}_{2} \mathrm{O}_{2}$ was detected by DAB staining at $24 \mathrm{~h}$ after inoculation. Expression of the defense genes was analyzed by qRT-PCR and relative expression was shown as folds of the transcript levels at different time points vs. the corresponding values at $0 \mathrm{~h}$ after inoculation. Data presented in $\mathbf{( B )}$ are the means \pm SD from three independent experiments and ${ }^{* *}$ above the columns indicate significant difference at $p<0.05$ level.

\section{SIRbohB is Required for Drought Stress Tolerance}

The involvement of Rbohs in abiotic stress has been documented recently (Kaur et al., 2014). We therefore examined whether SlRbohB has a function in the abiotic response by analyzing the effect of silencing of SlRbohB on drought stress tolerance. Under normal watering conditions, the TRV2-SlRbohBinfiltrated plants grew as well as the TRV2-GUS-infiltrated plants (Figure 8A). However, the TRV2-SlRbohB-infiltrated plants displayed significant wilting symptom and their leaves began to curl after 10 days of drought stress treatment, whereas the TRV2-GUS-infiltrated plants did not show any stress symptoms (Figure 8A). The rate of water loss in the leaves of the TRV2-SlRbohB-infiltrated plants was $>45 \%$ greater than that in the leaves of the TRV2-GUS-infiltrated plants at 2 and $3 \mathrm{~h}$ after detachment (Figure 8B). This finding indicates that silencing of SlRbohB accelerated water loss in leaves. Under normal watering conditions, the expression level of the drought-upregulated stress-responsive gene SGN-U213276 (Gong et al., 2010) in the TRV2-SlRbohB-infiltrated plants was comparable to that in TRV2-GUS-infiltrated plants, while the expression level of $S G N-214777$, a drought-downregulated stress-responsive gene (Gong et al., 2010), was lower in the 


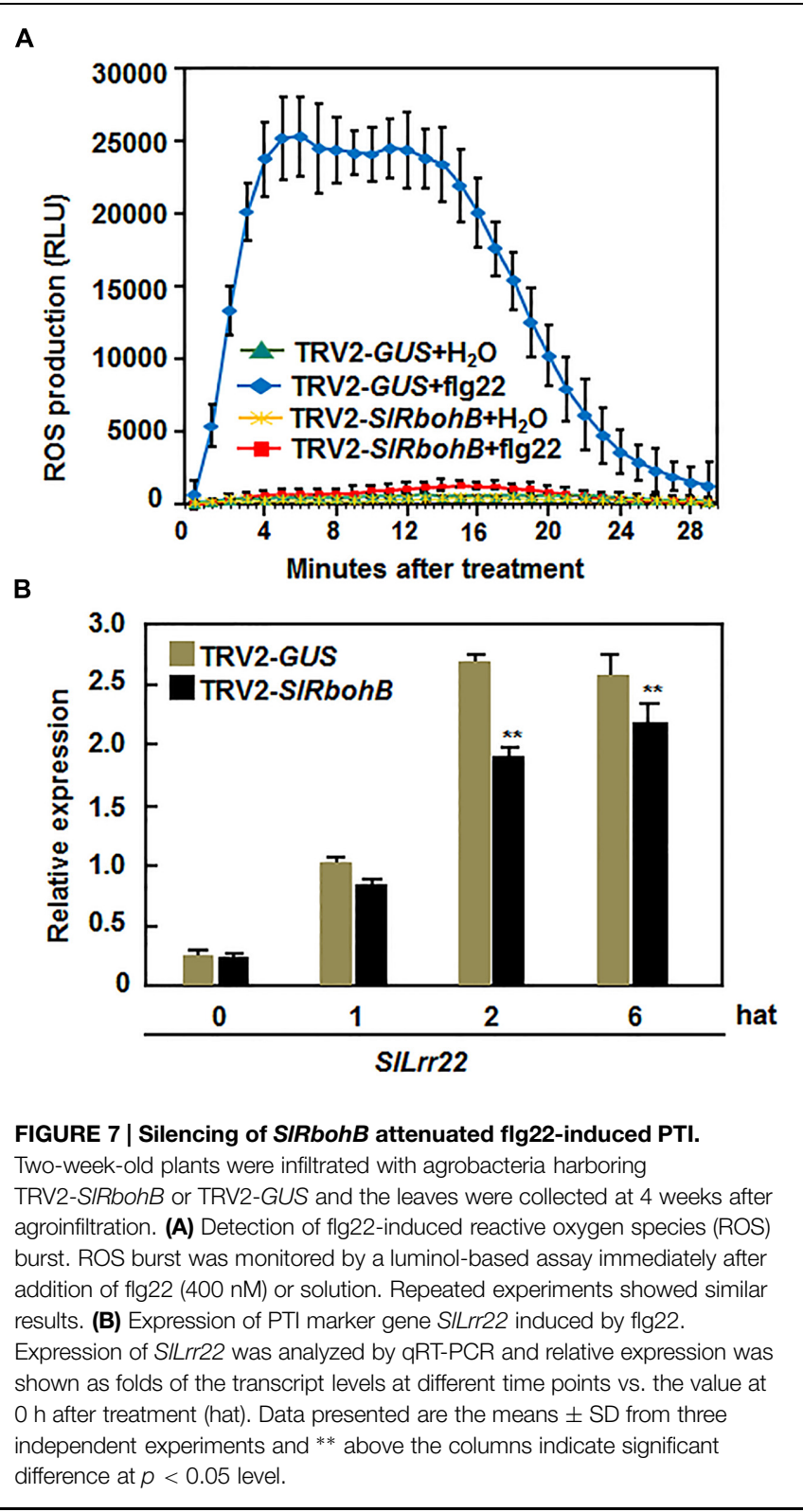

TRV2-SlRbohB-infiltrated plants than that in TRV2-GUSinfiltrated plants (Figure 8C). After drought stress treatment, the expression of SGN-213276 was induced in both TRV2-SlRbohBand TRV2-GUS-infiltrated plants (Figure 8C); however, the expression level of this gene in the TRV2-SlRbohB-infiltrated plants was lower than that in TRV2-GUS-infiltrated plants (Figure 8C). In contrast, the expression level of SGN-214777 in the TRV2-GUS-infiltrated plants was lower than that in TRV2SlRbohB-infiltrated plants under normal watering condition but was significantly reduced as compared with those in the TRV2-GUS-infiltrated plants after drought stress treatment (Figure 8C). These data indicate that silencing of SlRbohB resulted in reduced drought tolerance and thus SIRbohB has a positive function in regulating the drought stress response in tomato.

\section{Discussion}

In this study, we identified eight Rboh family members in tomato (Table 1). All SlRboh proteins share characteristic structural features in terms of the presence and organization of functional domains with previously identified plant Rboh proteins (Figure 1). Two of the SlRboh genes, SlRbohB (SlWfi1), and SlRbohG (SlRboh1), have been previously identified (Sagi et al., 2004) and shown to play roles in developmental processes, abiotic stress, and wounding responses (Sagi et al., 2004; Zhou et al., 2012, 2014; Xia et al., 2014). In the present study, we carried out a systemic VIGS-based functional analysis of the SIRboh family in stress responses. Our results demonstrate that SIRbohB plays important roles in resistance to $B$. cinerea and flg22-induced PTI and also functions in drought stress tolerance.

The activity of Rboh proteins may be regulated at both transcriptional and post-transcriptional levels. Rboh family members in various plants have been shown to be induced by wounding, pathogens, or different abiotic stress stimuli (Yoshioka et al., 2003; Proels et al., 2010; Suzuki et al., 2011; Chaouch et al., 2012; Ma et al., 2012; Montiel et al., 2012; Zhou et al., 2012; Pastor et al., 2013; Siddique et al., 2014; Xu et al., 2014). In the present study, we found that some of the tomato SlRboh genes are responsive to B. cinerea and Pst DC3000 (Figure 2). Among these pathogen-inducible SlRbohs, expression of SlRbohA, SlRbohB, and SlRbohC was induced by both of $B$. cinerea and Pst DC3000; whereas expression of SlRbohE, SlRbohF, and SlRbohG was induced only by B. cinerea but not by Pst DC3000 (Figure 2). The differential responsiveness of the SlRboh genes to pathogens implies that they play different roles in the response to biotic stress. The significance of the transcriptional regulation of Rbohs is supported by several observations that overexpression or silencing of Rboh genes resulted in altered resistance to a range of pathogens (Torres et al., 2002, 2005; Perchepied et al., 2010). Although expression of SlRbohA, SlRbohC, SlRbohE, SlRbohF, and SlRbohG was induced by $B$. cinerea (Figure 2A), our data indicate that these SIRbohs may not be involved in resistance to $B$. cinerea as silencing of each of these genes did not affect the resistance to $B$. cinerea (Figure 3 ). Alternatively, it is possible that post-translational modification is required for the enzyme activity of these SIRbohs after the expression is upregulated at the transcriptional level by pathogens or other stimuli. It was previously reported that post-translational regulation of Rboh activity is required for ROS production as overexpression of the Rboh gene did not result in constitutive ROS production (Torres et al., 2005; Kobayashi et al., 2012; Asai et al., 2013). Thus, further biochemical experiments are necessary to examine the possibility that post-translational modification is involved in regulating the activity of these SlRbohs, which are required to modulate the generation of ROS in tomato plants upon infection of $B$. cinerea.

It was suggested that Rboh-dependent ROS may contribute to help the necrotrophic pathogens establish colonization in plant tissues (Kobayashi et al., 2012). In our VIGS-based functional analyses, we found that silencing of $S l R b o h B$ resulted in reduced resistance against $B$. cinerea, as the SlRbohBsilenced plants exhibited severer severity of the disease and 
A

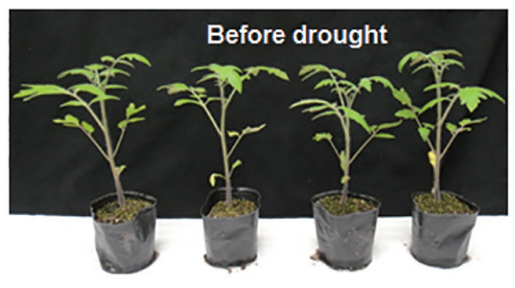

TRV2-GUS

TRV2-SIRbohB

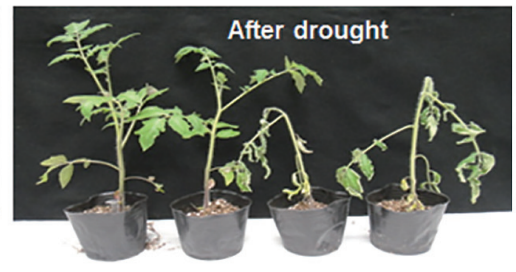

B
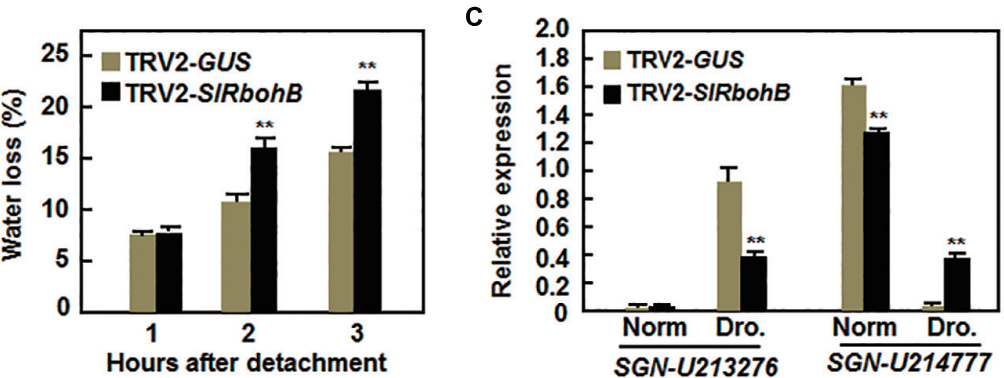

FIGURE 8 | Silencing of SIRbohB led to reduced drought tolerance. Two-week-old plants were infiltrated with agrobacteria harboring TRV2-SIRbohB or TRV2-GUS and the TRV2-SIRbohB- and TRV2-GUS-infiltrated plants were subjected to drought assays at 4 weeks after agroinfiltration. (A) Growth performance of the TRV2-SIRbohB- and TRV2-GUS-infiltrated plants under normal watering conditions (left) and at 10 days after drought stress treatment by withholding watering (right). (B) Rates of water loss in detached leaves of the
TRV2-SIRbohB- and TRV2-GUS-infiltrated plants. (C) Expression of drought-responsive genes. Leaf samples were collected from the TRV2-SIRbohB- and TRV2-GUS-infiltrated plants under normal (Norm) and drought (Dro.) conditions and relative expression was shown as folds of that of SIActin. Data presented in $\mathbf{( B , C )}$ are the means \pm SD from three independent experiments and ${ }^{* *}$ above the columns indicate significant difference at $p<0.05$ level. supported much in planta growth of $B$. cinerea as compared with the non-silenced plants (Figure 4). This is in contrast with observations that $N b R b o h B$-silenced $N$. benthamiana plants displayed increased resistance to $B$. cinerea (Asai and Yoshioka, 2009) and knockout of AtRbohD, the Arabidopsis homolog of SlRbohB, showed increased resistance to Fusarium oxysporum (Zhu et al., 2013). However, recent studies found that Rbohmediated ROS accumulation is not strictly correlated with disease susceptibility to B. cinerea. For example, treatment of Arabidopsis leaves with oligogalacturonides (OGs) elicited an AtRbohDdependent ROS burst and subsequently protected plants from attack by $B$. cinerea and the AtRbohD mutant plants exhibited an induction of defense genes and an increased resistance to B. cinerea after OG treatment (Galletti et al., 2008). Conversely, Rboh-generated ROS are thought to act as one of the earliest signaling events that mediate the activation of immune responses (Kaur et al., 2014). The fact that reduced resistance to $B$. cinerea in the $S l R b o h B$-silenced plants (Figure 4) is accompanied by a decrease in the $B$. cinerea-induced accumulation of ROS and the expression of defense genes (Figure 6) suggest that SlRbohBdependent generation of ROS should act to activate the defense response rather than promote pathogen infection. This is in agreement with previous observations concerning AtRbohD, which negatively regulates cell death and whose mutant plants reduced the accumulation of ROS induced by $B$. cinerea (Torres et al., 2005). However, whether silencing of SlRbohB, closely related to AtRbohD, has an effect on cell death and if so, the relationship between SlRbohB-regulated cell death and the reduced resistance to $B$. cinerea in $S l R b o h B$-silenced plants are open questions to be further investigated. Furthermore, we also found that the transient expression of SlRbohB in N. benthamiana conferred an enhanced resistance to $B$. cinerea (Figure 5). Taken together, these data demonstrate that SlRbohB functions as a positive regulator of the defense response to $B$. cinerea in tomato.

During PTI, the ROS burst is an early response that is believed to play important roles in the activation of the immune response (Zipfel et al., 2004; Nicaise et al., 2009). The involvement of Rbohs and Rboh-generated ROS in plant immune responses including PTI has been well established (Torres, 2010; Pastor et al., 2013). flg22, a well characterized PAMP, is perceived by SIFLS2 in tomato (Robatzek et al., 2007). Treatment with flg22 can not only induce globally the expression of defense genes and accumulation of ROS production but also protect plants from subsequent infection with virulent pathogens (Mueller et al., 2012). In the present study, we found that the flg22-induced ROS burst was greatly suppressed in $S l R b o h B$-silenced plants (Figure 7A). This is similar to the report that the flg22-induced ROS burst in Arabidopsis AtRbohD mutant plants was completely abolished (Zhang et al., 2007; Kadota et al., 2014; Li et al., 2014b). The flg22-induced expression of SlLrr22, a PTI marker gene in tomato (Taylor et al., 2012), was attenuated in the SlRbohB-silenced plants (Figure 7B). These experimental data demonstrate that, in addition to the function in resistance to $B$. cinerea, SIRbohB also play a role in regulating PTI in tomato. Recently, it was found that receptor-like cytoplasmic kinase BIK1, a component of the FLS2 immune receptor complex, directly phosphorylates AtRbohD in a calcium-independent manner to enhance ROS generation (Kadota et al., 2014; Li et al., 2014b). Conversely, mutation of 
the flg22 receptor AtFLS2 abolished the flg22-induced ROS burst and led to enhanced susceptibility to B. cinerea in Arabidopsis (Zipfel et al., 2004) and early accumulation of ROS was found to be correlated with resistance to $B$. cinerea in the ABA-deficient mutant sitens in tomato (Asselbergh et al., 2007). It is thus possible that the attenuated flg22-induced ROS burst is related to or even responsible for the reduced resistance to $B$. cinerea in SlRbohB-silenced plants. However, the mechanism of SIRbohB in PTI and the relationship between SlRbohB-mediated ROS burst and resistance to $B$. cinerea need to be investigated further.

Rbohs-generated ROS has been shown to regulate responses to various abiotic stresses such as wounding, light/radiation and ozone exposure, and salinity (Baxter et al., 2014). In Arabidopsis, AtRbohD- and AtRbohF-generated ROS is involved in stomatal closure in guard cells, the cold stress response and systemic acquired acclimation (Kwak et al., 2003; Kawarazaki et al., 2013; Suzuki et al., 2013). In the present study, we found that silencing of $S I R b o h B$ resulted in reduced drought tolerance (Figure 8A), indicating that SlRbohB also functions in drought stress tolerance in tomato. This hypothesis is supported by the observations that the detached leaves from the SlRbohBsilenced plants exhibited higher rate of water loss (Figure 8B) and altered expression of drought-responsive genes SGN-U213276 and $S G N-U 214777$, which were found to be upregulated or downregulated in drought stress, respectively (Gong et al., 2010), under stress condition (Figure 8C). Thus, it is possible that silncing of $S l R b o h B$ attenuated the drought stress response and thereby reduced drought tolerance in tomato. In addition, Rbohs have been shown to be involved in root system development (Foreman et al., 2003; Kwak et al., 2003; Jiao et al., 2013), which may affect the capacity of plants to take up water from the soil. However, silencing of SlRbohB did not affect the root system in SlRbohB-silenced plants (data not shown), indicating a limited role for the root system in SIRbohB-regulated drought tolerance.

\section{References}

AbuQamar, S., Chai, M. F., Luo, H., Song, F., and Mengiste, T. (2008). Tomato protein kinase $1 \mathrm{~b}$ mediates signaling of plant responses to necrotrophic fungi and insect herbivory. Plant Cell 20, 1964-1983. doi: 10.1105/tpc.108. 059477

Apel, K., and Hirt, H. (2004). Reactive oxygen species: metabolism, oxidative stress, and signal transduction. Annu. Rev. Plant Biol. 55, 373-399. doi: 10.1146/annurev.arplant.55.031903.141701

Arthikala, M. K., Montiel, J., Nava, N., Santana, O., Sanchez-Lopez, R., Cardenas, L., et al. (2013). PvRbohB negatively regulates Rhizophagus irregularis colonization in Phaseolus vulgaris. Plant Cell Physiol. 54, 1391-1402. doi: 10.1093/pcp/pct089

Arthikala, M. K., Sanchez-Lopez, R., Nava, N., Santana, O., Cardenas, L., and Quinto, C. (2014). RbohB, a Phaseolus vulgaris NADPH oxidase gene, enhances symbiosome number, bacteroid size, and nitrogen fixation in nodules and impairs mycorrhizal colonization. New Phytol. 202, 886-900. doi: $10.1111 /$ nph.12714

Asai, S., Ichikawa, T., Nomura, H., Kobayashi, M., Kamiyoshihara, Y., Mori, H., et al. (2013). The variable domain of a plant calcium-dependent protein kinase (CDPK) confers subcellular localization and substrate recognition for NADPH oxidase. J. Biol. Chem. 288, 14332-14340. doi: 10.1074/jbc.M112. 448910

\section{Conclusion}

Rbohs mediate the generation of ROS, thereby regulating a diverse range of biological processes in plants. The present study focused on the function of the SIRboh family in biotic and abiotic stress responses in tomato. The results from VIGS- and transient expression-based functional analyses clearly demonstrate that, in addition to the previously reported involvement in development and wounding response (Sagi et al., 2004), SlRbohB positively regulates the resistance to $B$. cinerea, flg22-induced PTI, and drought stress tolerance in tomato. Further work in decoding downstream signaling will help elucidate the molecular and physiological mechanisms by which SIRbohB regulates biotic and abiotic stress responses.

\section{Author Contributions}

XL, HZ, LT, LH, and SL carried out most of the experiments. DL performed bioinformatics analysis. DL, XL, and FS designed the experiments. FS and DL wrote the paper. All authors read and approved the final manuscript.

\section{Acknowledgment}

This work was supported by the National High-Tech R \& D Program (No. 2012AA101504 and 2012AA101505) and the National Basic Research Program of China (2009CB119005).

\section{Supplementary Material}

The Supplementary Material for this article can be found online at: http://journal.frontiersin.org/article/10.3389/fpls.2015.00463

Asai, S., Ohta, K., and Yoshioka, H. (2008). MAPK signaling regulates nitric oxide and NADPH oxidase-dependent oxidative bursts in Nicotiana benthamiana. Plant Cell 20, 1390-1406. doi: 10.1105/tpc.107.055855

Asai, S., and Yoshioka, H. (2009). Nitric oxide as a partner of reactive oxygen species participates in disease resistance to nectrotophic pathogen Botrytis cinerea in Nicotiana benthamiana. Mol. Plant Microbe Interact. 22, 619-629. doi: 10.1094/MPMI-22-6-0619

Asselbergh, B., Curvers, K., Franca, S. C., Audenaert, K., Vuylsteke, M., Van Breusegem, F., et al. (2007). Resistance to Botrytis cinerea in sitiens, an abscisic acid-deficient tomato mutant, involves timely production of hydrogen peroxide and cell wall modifications in the epidermis. Plant Physiol. 144, 1863-1877. doi: 10.1104/pp.107.099226

Baxter, A., Mittler, R., and Suzuki, N. (2014). ROS as key players in plant stress signalling. J. Exp. Bot. 65, 1229-1240. doi: 10.1093/jxb/ert375

Bright, J., Desikan, R., Hancock, J. T., Weir, I. S., and Neill, S. J. (2006). ABAinduced NO generation and stomatal closure in Arabidopsis are dependent on H2O2 synthesis. Plant J. 45, 113-122. doi: 10.1111/j.1365-313X.2005.02615.x

Canton, J., and Grinstein, S. (2014). Priming and activation of NADPH oxidases in plants and animals. Trends Immunol. 35, 405-407. doi: 10.1016/j.it.2014.07.007

Chakravarthy, S., Velasquez, A. C., Ekengren, S. K., Collmer, A., and Martin, G. B. (2010). Identification of Nicotiana benthamiana genes involved in pathogenassociated molecular pattern-triggered immunity. Mol. Plant-Microbe Interact. 23, 715-726. doi: 10.1094/MPMI-23-6-0715 
Chaouch, S., Queval, G., and Noctor, G. (2012). AtRbohF is a crucial modulator of defence-associated metabolism and a key actor in the interplay between intracellular oxidative stress and pathogenesis responses in Arabidopsis. Plant J. 69, 613-627. doi: 10.1111/j.1365-313X.2011.04816.x

Demidchik, V., Shang, Z., Shin, R., Thompson, E., Rubio, L., Laohavisit, A., et al. (2009). Plant extracellular ATP signalling by plasma membrane NADPH oxidase and $\mathrm{Ca}^{2+}$ channels. Plant J. 58, 903-913. doi: 10.1111/j.1365313X.2009.03830.x

Desikan, R., Last, K., Harrett-Williams, R., Tagliavia, C., Harter, K., Hooley, R., et al. (2006). Ethylene-induced stomatal closure in Arabidopsis occurs via AtrbohF-mediated hydrogen peroxide synthesis. Plant J. 47, 907-916. doi: 10.1111/j.1365-313X.2006.02842.x

Dubiella, U., Seybold, H., Durian, G., Komander, E., Lassig, R., Witte, C. P., et al. (2013). Calcium-dependent protein kinase/NADPH oxidase activation circuit is required for rapid defense signal propagation. Proc. Natl. Acad. Sci. U.S.A. 110, 8744-8749. doi: 10.1073/pnas.1221294110

Edgar, R. C. (2004). MUSCLE: multiple sequence alignment with high accuracy and high throughput. Nucleic Acids Res. 32, 1792-1797. doi: 10.1093/nar/gkh340

Foley, R. C., Gleason, C. A., Anderson, J. P., Hamann, T., and Singh, K. B. (2013). Genetic and genomic analysis of Rhizoctonia solani interactions with Arabidopsis; evidence of resistance mediated through NADPH oxidases. PLoS ONE 8:e56814. doi: 10.1371/journal.pone.0056814

Foreman, J., Demidchik, V., Bothwell, J. H., Mylona, P., Miedema, H., Torres, M. A., et al. (2003). Reactive oxygen species produced by NADPH oxidase regulate plant cell growth. Nature 422, 442-446. doi: 10.1038/ nature01485

Galletti, R., Denoux, C., Gambetta, S., Dewdney, J., Ausubel, F. M., De Lorenzo, G., et al. (2008). The AtrbohD-mediated oxidative burst elicited by oligogalacturonides in Arabidopsis is dispensable for the activation of defense responses effective against Botrytis cinerea. Plant Physiol. 148, 1695-1706. doi: 10.1104/pp.108.127845

Gong, P., Zhang, J., Li, H., Yang, C., Zhang, C., Zhang, X., et al. (2010). Transcriptional profiles of drought-responsive genes in modulating transcription signal transduction, and biochemical pathways in tomato. J. Exp. Bot. 61, 3563-3575. doi: 10.1093/jxb/erq167

Jiao, Y., Sun, L., Song, Y., Wang, L., Liu, L., Zhang, L., et al. (2013). AtrbohD and AtrbohF positively regulate abscisic acid-inhibited primary root growth by affecting $\mathrm{Ca}^{2+}$ signalling and auxin response of roots in Arabidopsis. J. Exp. Bot. 64, 4183-4192. doi: 10.1093/jxb/ert228

Jones, M. A., Raymond, M. J., Yang, Z., and Smirnoff, N. (2007). NADPH oxidasedependent reactive oxygen species formation required for root hair growth depends on ROP GTPase. J. Exp. Bot. 58, 1261-1270. doi: 10.1093/jxb/erl279

Kadota, Y., Sklenar, J., Derbyshire, P., Stransfeld, L., Asai, S., Ntoukakis, V., et al. (2014). Direct regulation of the NADPH oxidase RBOHD by the PRRassociated kinase BIK1 during plant immunity. Mol. Cell 54, 43-55. doi: 10.1016/j.molcel.2014.02.021

Kaur, G., Sharma, A., Guruprasad, K., and Pati, P. K. (2014). Versatile roles of plant NADPH oxidases and emerging concepts. Biotechnol. Adv. 32, 551-563. doi: 10.1016/j.biotechadv.2014.02.002

Kawarazaki, T., Kimura, S., Iizuka, A., Hanamata, S., Nibori, H., Michikawa, M., et al. (2013). A low temperature-inducible protein AtSRC2 enhances the ROSproducing activity of NADPH oxidase AtRbohF. Biochim. Biophys. Acta 1833, 2775-2780. doi: 10.1016/j.bbamcr.2013.06.024

Kaya, H., Nakajima, R., Iwano, M., Kanaoka, M. M., Kimura, S., Takeda, S., et al. (2014). $\mathrm{Ca}^{2+}$-activated reactive oxygen species production by Arabidopsis $\mathrm{RbohH}$ and RbohJ is essential for proper pollen tube tip growth. Plant Cell 26, 1069-1080. doi: 10.1105/tpc.113.120642

Kimura, S., Kaya, H., Kawarazaki, T., Hiraoka, G., Senzaki, E., Michikawa, M., et al. (2012). Protein phosphorylation is a prerequisite for the $\mathrm{Ca}^{2+}$-dependent activation of Arabidopsis NADPH oxidases and may function as a trigger for the positive feedback regulation of $\mathrm{Ca}^{2+}$ and reactive oxygen species. Biochim. Biophys. Acta 1823, 398-405. doi: 10.1016/j.bbamcr.2011.09.011

Kobayashi, M., Ohura, I., Kawakita, K., Yokota, N., Fujiwara, M., Shimamoto, K., et al. (2007). Calcium-dependent protein kinases regulate the production of reactive oxygen species by potato NADPH oxidase. Plant Cell 19, 1065-1080. doi: $10.1105 /$ tpc.106.048884

Kobayashi, M., Yoshioka, M., Asai, S., Nomura, H., Kuchimura, K., Mori, H., et al. (2012). StCDPK5 confers resistance to late blight pathogen but increases susceptibility to early blight pathogen in potato via reactive oxygen species burst. New Phytol. 196, 223-237. doi: 10.1111/j.1469-8137.2012.04226.x

Kwak, J. M., Mori, I. C., Pei, Z. M., Leonhardt, N., Torres, M. A., Dangl, J. L., et al. (2003). NADPH oxidase AtrbohD and AtrbohF genes function in ROS-dependent ABA signaling in Arabidopsis. EMBO J. 22, 2623-2633. doi: 10.1093/emboj/cdg277

Lassig, R., Gutermuth, T., Bey, T. D., Konrad, K. R., and Romeis, T. (2014) Pollen tube $\mathrm{NAD}(\mathrm{P}) \mathrm{H}$ oxidases act as a speed control to dampen growth rate oscillations during polarized cell growth. Plant J. 78, 94-106. doi: 10.1111/tpj.12452

Lherminier, J., Elmayan, T., Fromentin, J., Elaraqui, K. T., Vesa, S., Morel, J., et al. (2009). NADPH oxidase-mediated reactive oxygen species production: subcellular localization and reassessment of its role in plant defense. Mol. Plant Microbe Interact. 22, 868-881. doi: 10.1094/MPMI-22-7-0868

Li, N., Sun, L., Zhang, L., Song, Y., Hu, P., Li, C., et al. (2015). AtrbohD and AtrbohF negatively regulate lateral root development by changing the localized accumulation of superoxide in primary roots of Arabidopsis. Planta 241, 591602. doi: 10.1007/s00425-014-2204-1

Li, X., Zhang, Y., Huang, L., Ouyang, Z., Hong, Y., Zhang, H., et al. (2014a). Tomato SIMKK2 and SIMKK4 contribute to disease resistance against Botrytis cinerea. BMC Plant Biol. 14:166. doi: 10.1186/1471-2229-14-166

Li, L., Li, M., Yu, L., Zhou, Z., Liang, X., Liu, Z., et al. (2014b). The FLS2-associated kinase BIK1 directly phosphorylates the NADPH oxidase RbohD to control plant immunity. Cell Host Microbe 15, 329-338. doi: 10.1016/j.chom.2014.02.009

Liu, B., Hong, Y. B., Zhang, Y. F., Li, X. H., Huang, L., Zhang, H. J., et al. (2014). Tomato WRKY transcriptional factor SIDRW1 is required for disease resistance against Botrytis cinerea and tolerance to oxidative stress. Plant Sci. 227, 145-156. doi: 10.1016/j.plantsci.2014.08.001

Liu, Y., Schiff, M., and Dinesh-Kumar, S. P. (2002). Virus-induced gene silencing in tomato. Plant J. 31, 777-786. doi: 10.1046/j.1365-313X.2002.01394.x

Livak, K. J., and Schmittgen, T. D. (2001). Analysis of relative gene expression data using real-time quantitative PCR and the 2(-Delta Delta C(T)). Methods 25, 402-408. doi: 10.1006/meth.2001.1262

Ma, L., Zhang, H., Sun, L., Jiao, Y., Zhang, G., Miao, C., et al. (2012). NADPH oxidase AtrbohD and AtrbohF function in ROS-dependent regulation of $\mathrm{Na}^{+} / \mathrm{K}^{+}$homeostasis in Arabidopsis under salt stress. J. Exp. Bot. 63, 305-317. doi: $10.1093 /$ jxb/err280

Macpherson, N., Takeda, S., Shang, Z., Dark, A., Mortimer, J. C., Brownlee, C., et al. (2008). NADPH oxidase involvement in cellular integrity. Planta 227, 1415-1418. doi: 10.1007/s00425-008-0716-2

Marino, D., Andrio, E., Danchin, E. G., Oger, E., Gucciardo, S., Lambert, A., et al. (2011). A Medicago truncatula NADPH oxidase is involved in symbiotic nodule functioning. New Phytol. 189, 580-592. doi: 10.1111/j.1469-8137.2010.03509.x

Marino, D., Dunand, C., Puppo, A., and Pauly, N. (2012). A burst of plant NADPH oxidases. Trends Plant Sci. 17, 9-15. doi: 10.1016/j.tplants.2011.10.001

Maruta, T., Inoue, T., Tamoi, M., Yabuta, Y., Yoshimura, K., Ishikawa, T., et al. (2011). Arabidopsis NADPH oxidases, AtrbohD and AtrbohF, are essential for jasmonic acid-induced expression of genes regulated by MYC2 transcription factor. Plant Sci. 180, 655-660. doi: 10.1016/j.plantsci.2011.01.014

Miller, G., Schlauch, K., Tam, R., Cortes, D., Torres, M. A., Shulaev, V., et al. (2009). The plant NADPH oxidase RBOHD mediates rapid systemic signaling in response to diverse stimuli. Sci. Signal. 2, ra45. doi: 10.1126/scisignal.2000448

Miller, M. A., Pfeiffer, W., and Schwartz, T. (2010). "Creating the CIPRES science gateway for inference of large phylogenetic trees," in Proceedings of the Gateway Computing Environments Workshop (GCE), New Orleans, LA, 1-8. doi: 10.1109/GCE.2010.5676129

Mittler, R., Vanderauwera, S., Suzuki, N., Miller, G., Tognetti, V. B., Vandepoele, K., et al. (2011). ROS signaling: the new wave? Trends Plant Sci. 16, 300-309. doi: 10.1016/j.tplants.2011.03.007

Montiel, J., Nava, N., Cardenas, L., Sanchez-Lopez, R., Arthikala, M. K., Santana, O., et al. (2012). A Phaseolus vulgaris NADPH oxidase gene is required for root infection by Rhizobia. Plant Cell Physiol. 53, 1751-1767. doi: $10.1093 / \mathrm{pcp} / \mathrm{pcs} 120$

Mueller, K., Bittel, P., Chinchilla, D., Jehle, A. K., Albert, M., Boller, T., et al. (2012). Chimeric FLS2 receptors reveal the basis for differential flagellin perception in Arabidopsis and tomato. Plant Cell 24, 2213-2224. doi: 10.1105/tpc.112. 096073 
Müller, K., Carstens, A. C., Linkies, A., Torres, M. A., and Leubner-Metzger, G. (2009). The NADPH-oxidase AtrbohB plays a role in Arabidopsis seed afterripening. New Phytol. 184, 885-897. doi: 10.1111/j.1469-8137.2009.03005.x

Muller, K., Linkies, A., Leubner-Metzger, G., and Kermode, A. R. (2012). Role of a respiratory burst oxidase of Lepidium sativum (cress) seedlings in root development and auxin signalling. J. Exp. Bot. 63, 6325-6334. doi: $10.1093 /$ jxb/ers 284

Nathan, C., and Cunningham-Bussel, A. (2013). Beyond oxidative stress: an immunologist's guide to reactive oxygen species. Nat. Rev. Immunol. 13, 349361. doi: 10.1038/nri3423

Nestler, J., Liu, S., Wen, T. J., Paschold, A., Marcon, C., Tang, H. M., et al. (2014). Roothairless5, which functions in maize (Zea mays L.) root hair initiation and elongation encodes a monocot-specific NADPH oxidase. Plant J. 79, 729-740. doi: $10.1111 /$ tpj. 12578

Nicaise, V., Roux, M., and Zipfel, C. (2009). Recent advances in PAMP-triggered immunity against bacteria: pattern recognition receptors watch over and raise the alarm. Plant Physiol. 150, 1638-1647. doi: 10.1104/pp.109.139709

Noirot, E., Der, C., Lherminier, J., Robert, F., Moricova, P., Kieu, K., et al. (2014). Dynamic changes in the subcellular distribution of the tobacco ROS-producing enzyme RBOHD in response to the oomycete elicitor cryptogein. J. Exp. Bot. 65, 5011-5022. doi: 10.1093/jxb/eru265

Nozaki, M., Kita, K., Kodaira, T., and Ishikawa, A. (2013). AtRbohF contributes to non-host resistance to Magnaporthe oryzae in Arabidopsis. Biosci. Biotechnol. Biochem. 77, 1323-1325. doi: 10.1271/bbb.130092

Oda, T., Hashimoto, H., Kuwabara, N., Akashi, S., Hayashi, K., Kojima, C., et al. (2010). Structure of the N-terminal regulatory domain of a plant NADPH oxidase and its functional implications. J. Biol. Chem. 285, 1435-1445. doi: 10.1074/jbc.M109.058909

Ogasawara, Y., Kaya, H., Hiraoka, G., Yumoto, F., Kimura, S., Kadota, Y., et al. (2008). Synergistic activation of the Arabidopsis NADPH oxidase AtrbohD by $\mathrm{Ca}^{2+}$ and phosphorylation. J. Biol. Chem. 283, 8885-8892. doi: 10.1074/jbc.M708106200

Pastor, V., Luna, E., Ton, J., Cerezo, M., García-Agustín, P., and Flors, V. (2013). Fine tuning of reactive oxygen species homeostasis regulates primed immune responses in Arabidopsis. Mol. Plant Microbe Interact. 26, 1334-1344. doi: 10.1094/MPMI-04-13-0117-R

Perchepied, L., Balague, C., Riou, C., Claudel-Renard, C., Riviere, N., GrezesBesset, B., et al. (2010). Nitric oxide participates in the complex interplay of defense-related signaling pathways controlling disease resistance to Sclerotinia sclerotiorum in Arabidopsis thaliana. Mol. Plant Microbe Interact. 23, 846-860. doi: 10.1094/MPMI-23-7-0846

Pogány, M., Von Rad, U., Grün, S., Dongó, A., Pintye, A., Simoneau, P., et al. (2009). Dual roles of reactive oxygen species and NADPH oxidase RBOHD in an Arabidopsis-Alternaria pathosystem. Plant Physiol. 151, 1459-1475. doi: 10.1104/pp.109.141994

Proels, R. K., Oberhollenzer, K., Pathuri, I. P., Hensel, G., Kumlehn, J., and Huckelhoven, R. (2010). RBOHF2 of barley is required for normal development of penetration resistance to the parasitic fungus Blumeria graminis $\mathrm{f}$. sp. hordei. Mol. Plant Microbe Interact. 23, 1143-1150. doi: 10.1094/MPMI-23-9-1143

Robatzek, S., Bittel, P., Chinchilla, D., Köchner, P., Felix, G., Shiu, S. -H., et al. (2007). Molecular identification and characterization of the tomato flagellin receptor LeFLS2, an orthologue of Arabidopsis FLS2 exhibiting characteristically different perception specificities. Plant Mol. Biol. 64, 539-547. doi: 10.1007/s11103-007-9173-8

Sagi, M., Davydov, O., Orazova, S., Yesbergenova, Z., Ophir, R., Stratmann, J. W., et al. (2004). Plant respiratory burst oxidase homologs impinge on wound responsiveness and development in Lycopersicon esculentum. Plant Cell 16, 616-628. doi: 10.1105/tpc.019398

Sagi, M., and Fluhr, R. (2006). Production of reactive oxygen species by plant NADPH oxidases. Plant Physiol. 141, 336-340. doi: 10.1104/pp.106. 078089

Siddique, S., Matera, C., Radakovic, Z. S., Hasan, M. S., Gutbrod, P., Rozanska, E., et al. (2014). Parasitic worms stimulate host NADPH oxidases to produce reactive oxygen species that limit plant cell death and promote infection. Sci. Signal. 7, ra33. doi: 10.1126/scisignal.2004777

Simon-Plas, F., Elmayan, T., and Blein, J. P. (2002). The plasma membrane oxidase NtrbohD is responsible for AOS production in elicited tobacco cells. Plant J. 31, 137-147. doi: 10.1046/j.1365-313X.2002.01342.x
Sirichandra, C., Gu, D., Hu, H. C., Davanture, M., Lee, S., Djaoui, M., et al. (2009). Phosphorylation of the Arabidopsis AtrbohF NADPH oxidase by OST1 protein kinase. FEBS Lett. 583, 2982-2986. doi: 10.1016/j.febslet.2009.08.033

Song, C. J., Steinebrunner, I., Wang, X., Stout, S. C., and Roux, S. J. (2006). Extracellular ATP induces the accumulation of superoxide via NADPH oxidases in Arabidopsis. Plant Physiol. 140, 1222-1232. doi: 10.1104/pp.105.073072

Stamatakis, A. (2006). RAxML-VI-HPC: maximum likelihood-based phylogenetic analyses with thousands of taxa and mixed models. Bioinformatics 22, 26882690. doi: 10.1093/bioinformatics/btl446

Stamatakis, A., Hoover, P., and Rougemont, J. (2008). A rapid bootstrap algorithm for the RAxML Web servers. Syst. Biol. 57, 758-771. doi: $10.1080 / 10635150802429642$

Suzuki, N., Miller, G., Morales, J., Shulaev, V., Torres, M. A., and Mittler, R. (2011). Respiratory burst oxidases: the engines of ROS signaling. Curr. Opin. Plant Biol. 14, 691-699. doi: 10.1016/j.pbi.2011.07.014

Suzuki, N., Miller, G., Salazar, C., Mondal, H. A., Shulaev, E., Cortes, D. F., et al. (2013). Temporal-spatial interaction between reactive oxygen species and abscisic acid regulates rapid systemic acclimation in plants. Plant Cell 25, 3553-3569. doi: 10.1105/tpc.113.114595

Takahashi, S., Kimura, S., Kaya, H., Iizuka, A., Wong, H. L., Shimamoto, K., et al. (2012). Reactive oxygen species production and activation mechanism of the rice NADPH oxidase OsRbohB. J. Biochem. 152, 37-43. doi: 10.1093/jb/mvs044

Taylor, K. W., Kim, J. G., Su, X. B., Aakre, C. D., Roden, J. A., Adams, C. M., et al. (2012). Tomato TFT1 is required for PAMP-triggered immunity and mutations that prevent T3S effector XopN from binding to TFT1 attenuate Xanthomonas virulence. PLoS Pathog. 8:e1002768. doi: 10.1371/journal.ppat.1002768

Thordal-Christensen, H., Zhang, Z. G., Wei, Y. D., and Collinge, D. B. (1997). Subcellular localization of $\mathrm{H} 2 \mathrm{O} 2$ in plants. $\mathrm{H} 2 \mathrm{O} 2$ accumulation in papillae and hypersensitive response during the barley-powdery mildew interaction. Plant J. 11, 1187-1194. doi: 10.1046/j.1365-313X.1997.11061187.x

Torres, M. A. (2010). ROS in biotic interactions. Physiol. Plant. 138, 414-429. doi: 10.1111/j.1399-3054.2009.01326.x

Torres, M. A., Dangl, J. L., and Jones, J. D. (2002). Arabidopsis gp91phox homologues AtrbohD and AtrbohF are required for accumulation of reactive oxygen intermediates in the plant defense response. Proc. Natl. Acad. Sci. U.S.A. 99, 517-522. doi: 10.1073/pnas.012452499

Torres, M. A., Jones, J. D., and Dangl, J. L. (2005). Pathogen-induced, NADPH oxidase-derived reactive oxygen intermediates suppress spread of cell death in Arabidopsis thaliana. Nat. Genet. 37, 1130-1134. doi: 10.1038/ng1639

Torres, M. A., Jones, J. D., and Dangl, J. L. (2006). Reactive oxygen species signaling in response to pathogens. Plant Physiol. 141, 373-378. doi: 10.1104/pp.106.079467

Torres, M. A., Morales, J., Sanchez-Rodriguez, C., Molina, A., and Dangl, J. L. (2013). Functional interplay between Arabidopsis NADPH oxidases and heterotrimeric G protein. Mol. Plant Microbe Interact. 26, 686-694. doi: 10.1094/MPMI-10-12-0236-R

Tripathy, B. C., and Oelmuller, R. (2012). Reactive oxygen species generation and signaling in plants. Plant Signal. Behav. 7, 1621-1633. doi: 10.4161/psb. 22455

Trujillo, M., Altschmied, L., Schweizer, P., Kogel, K. H., and Huckelhoven, R. (2006). Respiratory burst oxidase homologue A of barley contributes to penetration by the powdery mildew fungus Blumeria graminis $\mathrm{f}$. $\mathrm{sp}$. hordei. J. Exp. Bot. 57, 3781-3791. doi: 10.1093/jxb/erl191

Wong, H. L., Pinontoan, R., Hayashi, K., Tabata, R., Yaeno, T., Hasegawa, K., et al. (2007). Regulation of rice NADPH oxidase by binding of Rac GTPase to its N-terminal extension. Plant Cell 19, 4022-4034. doi: 10.1105/tpc.107.055624

Wu, J., Wang, L., Wunsche, H., and Baldwin, I. T. (2013). Narboh D, a respiratory burst oxidase homolog in Nicotiana attenuata, is required for late defense responses after herbivore attack. J. Integr. Plant Biol. 55, 187-198. doi: 10.1111/j.1744-7909.2012.01182.x

Xia, X. J., Gao, C. J., Song, L. X., Zhou, Y. H., Shi, K., and Yu, J. Q. (2014). Role of $\mathrm{H} 2 \mathrm{O} 2$ dynamics in brassinosteroid-induced stomatal closure and opening in Solanum lycopersicum. Plant Cell Environ. 37, 2036-2050. doi: $10.1111 /$ pce. 12275

Xie, H. T., Wan, Z. Y., Li, S., and Zhang, Y. (2014). Spatiotemporal production of reactive oxygen species by NADPH oxidase is critical for tapetal programmed cell death and pollen development in Arabidopsis. Plant Cell 26, 2007-2023. doi: $10.1105 /$ tpc. 114.125427 
Xie, Y. J., Xu, S., Han, B., Wu, M. Z., Yuan, X. X., Han, Y., et al. (2011). Evidence of Arabidopsis salt acclimation induced by up-regulation of HY1 and the regulatory role of RbohD-derived reactive oxygen species synthesis. Plant J. 66, 280-292. doi: 10.1111/j.1365-313X.2011.04488.x

Xing, T., Wang, X. J., Malik, K., and Miki, B. L. (2001). Ectopic expression of an Arabidopsis calmodulin-like domain protein kinase-enhanced NADPH oxidase activity and oxidative burst in tomato protoplasts. Mol. Plant Microbe Interact. 14, 1261-1264. doi: 10.1094/MPMI.2001.14.10.1261

Xu, J., Xie, J., Yan, C., Zou, X., Ren, D., and Zhang, S. (2014). A chemical genetic approach demonstrates that MPK3/MPK6 activation and NADPH oxidase-mediated oxidative burst are two independent signaling events in plant immunity. Plant J. 77, 222-234. doi: 10.1111/tpj.12382

Yoshioka, H., Asai, S., Yoshioka, M., and Kobayashi, M. (2009). Molecular mechanisms of generation for nitric oxide and reactive oxygen species, and role of the radical burst in plant immunity. Mol. Cells 28, 321-329. doi: 10.1007/s10059-009-0156-2

Yoshioka, H., Numata, N., Nakajima, K., Katou, S., Kawakita, K., Rowland, O., et al. (2003). Nicotiana benthamiana gp91phox homologs NbrbohA and NbrbohB participate in $\mathrm{H} 2 \mathrm{O} 2$ accumulation and resistance to Phytophthora infestans. Plant Cell 15, 706-718. doi: 10.1105/tpc.008680

Yun, B. W., Feechan, A., Yin, M., Saidi, N. B., Le Bihan, T., Yu, M., et al. (2011). $S$-nitrosylation of NADPH oxidase regulates cell death in plant immunity. Nature 478, 264-268. doi: 10.1038/nature10427

Zhang, J., Shao, F., Li, Y., Cui, H., Chen, L., Li, H., et al. (2007). A Pseudomonas syringae effector inactivates MAPKs to suppress PAMP-induced immunity in plants. Cell Host Microbe 1, 175-185. doi: 10.1016/j.chom.2007.03.006

Zhang, Y., Zhu, H., Zhang, Q., Li, M., Yan, M., Wang, R., et al. (2009). Phospholipase dalphal and phosphatidic acid regulate NADPH oxidase activity and production of reactive oxygen species in ABA-mediated stomatal closure in Arabidopsis. Plant Cell 21, 2357-2377. doi: 10.1105/tpc.108.062992

Zhou, J., Wang, J., Li, X., Xia, X. J., Zhou, Y. H., Shi, K., et al. (2014). H2O2 mediates the crosstalk of brassinosteroid and abscisic acid in tomato responses to heat and oxidative stresses. J. Exp. Bot. 65, 4371-4383. doi: 10.1093/jxb/ eru217

Zhou, J., Wang, J., Shi, K., Xia, X. J., Zhou, Y. H., and Yu, J. Q. (2012). Hydrogen peroxide is involved in the cold acclimation-induced chilling tolerance of tomato plants. Plant Physiol. Biochem. 60, 141-149. doi: 10.1016/j.plaphy.2012.07.010

Zhu, Q. H., Stephen, S., Kazan, K., Jin, G., Fan, L., Taylor, J., et al. (2013). Characterization of the defense transcriptome responsive to Fusarium oxysporum-infection in Arabidopsis using RNA-seq. Gene 512, 259-266. doi: 10.1016/j.gene.2012.10.036

Zipfel, C., Robatzek, S., Navarro, L., Oakeley, E. J., Jones, J. D., Felix, G., et al. (2004). Bacterial disease resistance in Arabidopsis through flagellin perception. Nature 428, 764-767. doi: 10.1038/nature02485

Conflict of Interest Statement: The authors declare that the research was conducted in the absence of any commercial or financial relationships that could be construed as a potential conflict of interest.

Copyright (c) 2015 Li, Zhang, Tian, Huang, Liu, Li and Song. This is an open-access article distributed under the terms of the Creative Commons Attribution License (CC BY). The use, distribution or reproduction in other forums is permitted, provided the original author(s) or licensor are credited and that the original publication in this journal is cited, in accordance with accepted academic practice. No use, distribution or reproduction is permitted which does not comply with these terms. 